\title{
NUMERICAL COMPUTATION OF ELECTRIC FIELDS IN PRESENCE OF CURVILINEAR INTERFACE BETWEEN CONDUCTIVE AND NON-CONDUCTIVE MEDIA
}

\begin{abstract}
Purpose. To elaborate a method of electric field numerical calculation in systems with curved boundaries between conductive and non-conductive media at final volume method usage and application of the rectangular grids. Methodology. At electric field calculation in quasi-stationary approximation, potential of the whole conductive object (rod) is constant. At final difference scheme writing, presence of the curved part of the boundary between conducting and non-conducting media has been taking into account as follows. It was supposed that curved section complements the closed loop on which integration of the solvable equation is done instead of a straight section which extends within a conducting medium. Usage of this approach allows taking into account square of the curved sections of the boundary and distance between surface of non-conductive medium and nearest nodes of the computational grid. Results. Dependence of the maximum electric field intensity on the height and radius of curvature peaks rods has been got with the help of calculations. As a result, a polynomial approximation for the analytical expression of the external electric field intensity, upon which application to the conductive object of a certain height and radius of curvature of its top, corona discharges will develop. References 13, figures 4.
\end{abstract}

Key words: rounded tops, curvilinear borders, finite volume method, calculated grid, electric field intensity.

Описаны принципы учета криволинейных границ раздела при использовании метода конечных объемов для расчета усиления электрического поля на вериинах проводящих стержней. С помощью проведенных расчетов получена зависимость максимальной напряженности электрического поля от высоты и радиуса скругления вериин стержней. В результате аппроксимации данной зависимости полиномом записано аналитическое выражение для напряженности внешнего электрического поля, при приложении которого к проводящему объекту определенной высоты и радиуса скругления вериины на нем развивается коронный разряд. Бібл. 13, рис. 4.

Ключевые слова: скругленные вершины, криволинейные границы, метод конечных объемов, расчетная сетка, напряженность электрического поля.

Introduction. In a number of practically important cases information on the distribution character and level of the maximum electric field (EF) intensity in the surrounding of tops of conductive rods is required. Application of systems containing conductive rods, is one of the possible ways of practical realization of metamaterials, which are widely spread in recent years [1]. Systems «rod - plane» are used in high-voltage pulse engineering for initiation discharges in different media [2]. This category of objects is elements of power facilities that can be modeled by peaks with rods of various shapes. In particular, information about the intensity of external electric field intensities, at application of which corona discharges arise is necessary.

Typically, tops of rods used in engineering are rounded. In calculating the EF in such systems problem of taking into account the curvature of the surface of tops of rods arises. The application in this case of the finite element method does not solve problem fully because the elements used (e.g., triangles) have corners, which means that when calculating the intensity of the EF will be too high compared with the actual values. Taking into account that the main part of the rod is straight for the calculation it is appropriate to apply the finite-difference methods [3]. However, it is known that using the rectangular calculated mesh to describe the EF in systems with curved surfaces leads to a significant increase in the calculated levels of the EF intensity that has no place in reality [4]. Moreover, utilization of the more fine mesh only worsens the situation. To solve this problem, different techniques, such as representation of derivatives in the form of polynomials, not finite differences, are used [5]. However, this approach is linked to the complexity of the problem, so advantaged of the finite-difference methods such as simplicity and possibility to take into account nonlinear parameters of media are lost. To calculate electromagnetic fields in such systems so-called conformal schemes are used providing averaging of material parameters of media in volumes of cells located at their curved interfaces [6]. Utilization of this approach to media which material parameters differ not more than in 5-10 times, gives very good results [6-8]. But if these values differ by several orders of magnitude (for example, at the location of conducting rod in the air), then, as it will be shown below, using this method is ineffective.

The goal of the work is development of a simplified method of incorporation of curved boundary interfaces between conductive and non-conductive media using the calculated rectangular meshes for calculating electromagnetic fields in the respective systems and the use of this method to assess by what radius should be rounded the sharp edges on objects that are in the area of electric fields impact, so that there are not there corona discharges on them.

(C) E.I. Sokol, M.M. Rezinkina, E.V. Sosina, O.G. Gryb 
Electric field modeling at presence of curvilinear interfaces. We consider the cases where the distance at which the EF is changing significantly exceed the typical size of the objects under consideration, so the calculation can be done in quasi-stationary approximation. The equations that describe the distribution of the EF, we obtain in the following manner. We write Maxwell equations in the form [9]:

$$
\operatorname{rot} \vec{H}=\gamma \vec{E}+\frac{\partial \vec{D}}{\partial t},
$$

where $\vec{H}, \vec{E}$ are the intensities of magnetic and electric fields, respectively; $\vec{D}=\varepsilon_{0} \varepsilon \vec{E} ; \varepsilon_{0}=0.885 \cdot 10^{-11} \mathrm{~F} / \mathrm{m}$; $\varepsilon$ is the relative electric permeability; $\gamma$ is the conductivity.

We use for the numerical calculation the finite volume method, at the use of which to the considered area the rectangular mesh is applied, and the solved differential equation is obtained by using conservation laws. To do this, we take the divergence of both parts of (1) taking into account that at the steady-state mode the second term on the right side is close to zero. Then we integrate the obtained equation by elementary cell volumes, into which the estimated region is divided, and use the substitution $\vec{E}=-\operatorname{grad} \varphi$ (where $\varphi$ is the electrical potential). Finally, we obtain:

$$
\oint_{S}-\frac{\partial \varphi}{\partial n} \cdot \gamma d s=0,
$$

where $S$ are the sides of the parallelepiped, which divide in half the distances between neighboring nodes; $n$ is the direction of the normal to the integration contour.

Let us describe in detail the calculation of the EF near the rounded tops of rods. When using the finite volume method nodes in which values of potentials are calculated should be placed at the boundaries between interfaces [8]. This allows take into account boundary conditions automatically, without setting their individual equations.

It is proposed to use this approach to obtain solutions for intensities of the EF near curved surfaces of interfaces. We take into account that the system considered includes conductive (rod) and non-conductive (air) media. Moreover, in the quasi-stationary case the conductive object potential can be considered as constant. We consider three-dimensional objects. First, we consider the case of rectilinear interfaces (see Fig. 1,a).

The section $Z=$ const of closed contour of the square $S$ by which of the solved equation (2) is carried out may be presented in the form of four planes perpendicular to components of the intensity of the EF: $S_{1 x}$ and $S_{2 x}$ in the direction parallel to the axis $O x$, and $S_{1 y}$ and $S_{2 y}$ - in the direction parallel to the axis $O y$.

This is possible because integration of (2) by the surfaces $S_{1 x}^{B}$ and $S_{2 x}^{B}$ which are located close to the interface between media on both sides of it, in this case, can be replaced by integration by $S_{1 x}$ and $S_{2 x}$.
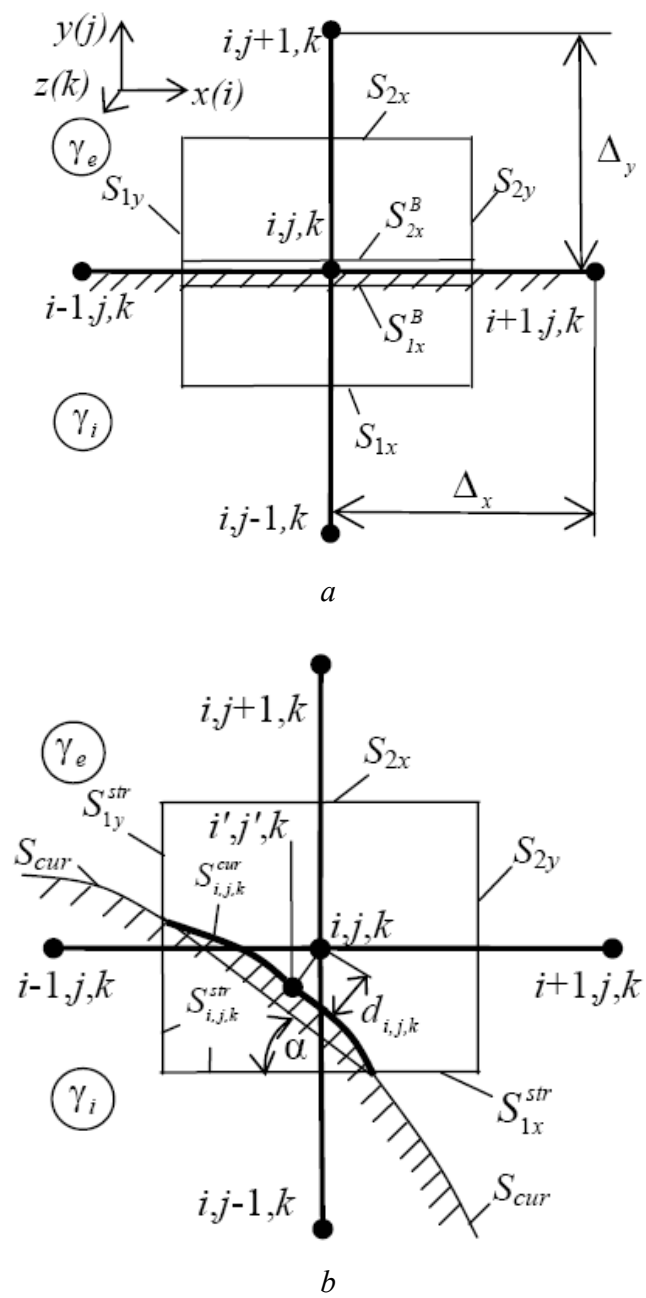

Fig. 1. Section of the calculating scheme by plane $Z=$ const in the case of rectilinear $(a)$ and curved $(b)$ interfaces

Such a substitution is possible because squares by which integration is carried out are equal: $S_{1 x}^{B}=S_{1 x}=$ $=S_{2 x}^{B}=S_{2 x r}=\Delta_{x}$ (where $\Delta_{x}$ is the step by space in the direction of the axis $O x$ ), and differential analogs of $y$ components of the EF intensities by both sides of the interface: $E y_{i, j}^{+}$and $E y_{i, j}^{-}$are determined by similar expressions: as derivatives at step back or step forward in the integration by $S_{1 x}^{B}$ and $S_{2 x}^{B}$ or as central derivatives at integration by $S_{1 x}$ and $S_{2 x}$ :

$$
\begin{aligned}
& E y_{i, j, k}^{+}=-\frac{\partial \varphi}{\partial y} \approx-\frac{\varphi_{i, j+1, k}-\varphi_{i, j, k}}{\Delta_{y}}, \\
& E y_{i, j}^{-}=-\frac{\partial \varphi}{\partial y} \approx-\frac{\varphi_{i, j, k}-\varphi_{i, j-1, k}}{\Delta_{y}},
\end{aligned}
$$

where $\varphi_{i, j, k}$ is the potential of the node $(i, j, k) ; \Delta_{y}$ is the step by space in the direction of the axis $O y$.

Let us write (2) in the differential form as a sum of integral by squares perpendicular to axis $O x$ and $O y$, taking into account that $\gamma_{e}<<\gamma_{i}$, and, therefore, $\gamma_{i}+\gamma_{e} \approx \gamma_{i}$ (where $\gamma_{i}, \gamma_{e}$ are conductivities of conductive and nonconductive media, respectively): 


$$
\begin{gathered}
\oint_{S}-\frac{\partial \varphi}{\partial n} \cdot \gamma d s \approx\left(F_{y 2}-F_{y 1}\right)+\left(F_{x 2}-F_{x 1}\right), \\
\text { де } F_{y 1}=-\gamma_{i} \cdot \frac{\varphi_{i, j, k}-\varphi_{i, j-1, k}}{\Delta_{y}} \cdot S_{1 x} \\
F_{y 2}=-\gamma_{e} \cdot \frac{\varphi_{i, j+1, k}-\varphi_{i, j, k}}{\Delta_{y}} \cdot S_{2 x} \\
F_{x 1} \approx-0.5 \cdot \gamma_{i} \cdot \frac{\varphi_{i, j, k}-\varphi_{i-1, j, k}}{\Delta_{x}} \cdot S_{1 y} \\
F_{x 2} \approx-0.5 \cdot \gamma_{i} \cdot \frac{\varphi_{i+1, j, k}-\varphi_{i, j, k}}{\Delta_{x}} \cdot S_{2 y} .
\end{gathered}
$$

We now consider $(i, j, k)$-th cell of the calculated system for which the $S$-contour of integration (2) includes a part $S_{i, j, k}^{c u r}$ of curved interface $S^{c u r}$ (see Fig. $1, b$ ). In order to take into account the presence of this area when writing the difference scheme, we assume that $S_{i, j, k}^{c u r}$ complements $S$ to closed contour instead of straight part $S_{i, j, k}^{s t r}$ that runs inside a conductive medium. As noted above, at the assumptions adopted the potential of all conductive object (rod) is the same, denoted by $U_{0}$. With that, the potential value in the nodes located inside a conductive medium, are obtained from the solution automatically when setting the level of potential $\varphi=U_{0}$ on the corresponding part of the outer boundary of the calculated area. Denote by En the EF intensity near the surface of the rod directed perpendicular to this surface. Since the potentials of all nodes located inside and on the surface of a conductive medium are the same, we assume that at the determination of $E n$ as a potential difference instead of nodes inside a conductive medium (in our case $(i-1, j, k)$ i $(i, j-1, k)-$ see. Fig. $1, b)$ can be used the node $\left(i^{\prime}, j^{\prime}, k^{\prime}\right)$ located on the interface at the place of contact of the perpendicular to the surface of the node $(i, j, k)$ :

$$
E n_{i, j, k}=-\frac{\partial \varphi}{\partial n} \approx-\frac{\varphi_{i, j, k}-\varphi_{i^{\prime}, j^{\prime}, k^{\prime}}}{d_{i, j, k}}=-\frac{\varphi_{i, j, k}-U_{0}}{d_{i, j, k}},
$$

where $d_{i, j, k}$ is the distance from the node $(i, j, k)$ to the curved interface.

Taking into account that the curvature in the direction of the axis $O z$ is small, we expand $E n$ on the $x$ and $y$ components, considering that the region is inclined to the horizontal at an angle $\alpha$ (see Fig. 1,b):

$$
\begin{aligned}
& -\frac{\partial \varphi}{\partial x}=E_{x} \approx E n \cdot \sin \alpha, \\
& -\frac{\partial \varphi}{\partial y}=E_{y} \approx E n \cdot \cos \alpha .
\end{aligned}
$$

Then the terms $F_{x 1}$ and $F_{y 1}$ in (4), for which the integration contour of equation (2) includes, in particular, curved planes of the interface, are written as:

$$
F_{x 1} \approx-\gamma_{e} \cdot \frac{\varphi_{i, j, k}-U_{0}}{d_{i, j, k}} \cdot\left\{\sin \alpha \cdot S_{1 y}^{s t r} \cdot\left[k_{x}+\left(1-k_{x}\right)\right]+k_{x} \cdot S_{i, j, k}^{c u r}\right\},(
$$

$F_{y 1} \approx-\gamma_{e} \cdot \frac{\varphi_{i, j, k}-U_{0}}{d_{i, j, k}} \cdot\left\{\cos \alpha \cdot S_{1 x}^{s t r} \cdot\left[k_{y} \cdot+\left(1-k_{y}\right)\right]+k_{y} \cdot S_{i, j, k}^{c u r}\right\},(12)$

where $k_{x}=\left\{\begin{array}{llc}0, & \text { if } & \alpha<\pi / 4 \\ 1, & \text { if } & \alpha>\pi / 4\end{array} ; \quad k_{y}=\left\{\begin{array}{llc}0, & \text { if } & \alpha>\pi / 4 \\ 1, & \text { if } & \alpha<\pi / 4\end{array} ;\right.\right.$

$S_{1 x}^{s t r}, S_{1 y}^{s t r}$ are the squares of straight parts of the contour

of integration $S ; S_{i, j, k}^{c u r}$ is the square of the curvilinear part $S$ (see Fig. 1,b).

In writing (11), (12) a simplified approach is used when it is assumed that when $\alpha>\pi / 4$ the part $S_{i, j, k}^{c u r}$ together with part $S_{1 y}$ or $S_{2 x}$ (in our case this is a part $S_{1 y}^{s t r}$ ) complements the part of the integration contour, parallel to the axis $O y$. If $\alpha<\pi / 4$, the curve $S_{i, j, k}^{c u r}$ is considered as a part of the integration contour, supplementing with parts $S_{1 x}$ or $S_{2 x}$ (in our case this is a segment $\left.S_{1 x}^{s t r}\right)$ a part of integration contour, parallel to axis $O x$. For the considered cell (see Fig. $1, b)$ terms $F_{y 2}$ and $F_{x 2}$ in (4) are determined by the expressions (6) and (8) as at their obtaining the curved parts are not included to the integration contour. Finding the length of the curved part $S_{i, j, k}^{c u r}$, straight segments $S_{1 x}^{s t r}, S_{1 y}^{s t r}$ as well as $d_{i, j, k}$ does not present significant difficulties and could be done, for example, numerically as a result of more fine dividing of cells that lie on the interface. And, by the proposed approach near-boundary cells located inside a conductive medium, are virtually displaced on it at the EF determination in non-conductive medium. This «displacement» does not affect the determination of EF inside a conductive medium because its potential is constant.

Generally, at the use of finite difference methods curvilinear boundary between media is replaced by a step approximating surface [6]. However, this causes the appearance at the calculation of local areas with increased intensity of the EF that really has no place [4]. Moreover, the level of EF intensity in the areas adjacent to the corners of rectangular cells approximating the curved surface will be greater, the smaller step of the spatial calculation mesh. The proposed method allowed tavoid this problem. Fig. 2 shows the EF intensity distributions on the conductive sphere surface areas that is in the homogeneous EF calculated by using the described approach (see the dotted curve 2).

Calculation is carried out at the step of the mesh $\Delta=0.02 \cdot R$ (where $R$ is the radius of the sphere). Levels of the EF intensity modulus in the $(i, j, k)$-th node are determined by calculated potentials in the nodes of the mesh as average values by the cell volume:

$$
|\vec{E}|=\sqrt{E x_{i, j, k}^{2}+E y_{i, j, k}^{2}+E z_{i, j, k}^{2}}
$$




$$
\begin{aligned}
\text { де } E x_{i, j, k} & \approx-0.5 \cdot\left(\frac{\varphi_{i+1, j, k}-\varphi_{i, j, k}}{\Delta_{x}}+\frac{\varphi_{i, j, k}-\varphi_{i-1, j, k}}{\Delta_{x}}\right) \\
E y_{i, j, k} & \approx-0.5 \cdot\left(\frac{\varphi_{i, j+1, k}-\varphi_{i, j, k}}{\Delta_{x}}+\frac{\varphi_{i, j, k}-\varphi_{i, j-1, k}}{\Delta_{y}}\right) \\
E z_{i, j, k} & \approx-0.5 \cdot\left(\frac{\varphi_{i+1, j, k+1}-\varphi_{i, j, k}}{\Delta_{z}}+\frac{\varphi_{i, j, k}-\varphi_{i, j, k-1}}{\Delta_{z}}\right)
\end{aligned}
$$

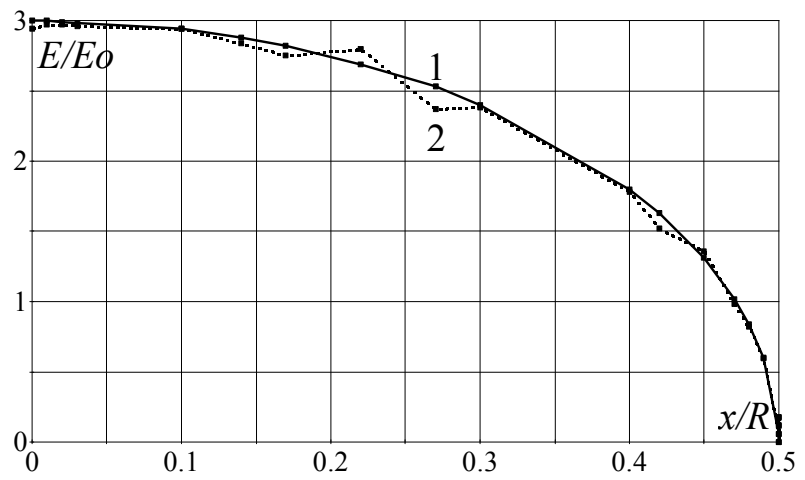

Fig. 2. Calculated EF intensity distributions on the surface of the conductive sphere of radius $R$ in the homogeneous external EF of intensity $E_{0}$ : 1 - analytical solution, 2 - solution by proposed method

For comparison, in the same figure (see curve 1) the analytical solution for the conductive sphere in a homogeneous external EF is shown [10]. As can be seen from a comparison of curves 1 and 2, the relative differences in the intensity of the EF for these two cases are very small, they do not exceed $3 \%$. As the calculations with the same step in space demonstrate, at the step approximation of the interface the relative differences reach almost $30 \%$.

As noted above, to address this problem, a number of methods are used [4-6]. They are rather bulky, which greatly complicates their practical implementation in contrast to the described approach, not associated with the introduction of non-orthogonal mesh or increase the order of approximation of derivatives.

Thus, the use of the proposed approach allows take into account the square of curved sections of interfaces as well as the distances between the non-conductive medium between arranged in near-boundary medium nodes of computational mesh and the interface. This is possible due to the use to obtain the solved equations of the conservation law (in this case, for charge) through the integration of initial differential equations on contours of cells of the calculated taking into account configuration of the interface within each cell.

As the calculations are shown, the EF intensity values obtained using the conformal scheme [6] coincide fully with those obtained using the step approximation. This is because with such a large difference between the values of conductivity of conductive and non-conductive media (more than 5-6 orders) the reduce by several times of the equivalent conductivity of cells located at the interface and containing conductive and non-conductive medium practically does not influence impact on calculated levels of potentials.

Calculation of $\mathrm{EF}$ in the neighborhood of a cylindrical wire rod. The approached described above permit to perform the calculation of the electric field in the neighborhood of the conductive cylindrical rod that is in the vertically directed external EF of intensity $E_{0}$. The calculated system that includes a conductive cylindrical rod with rounded top 1 is shown in Fig. 3.

Analytical solutions for EF intensity in systems containing cylindrical rods with rounded tops are absent. To find the distribution of EF we use the numerical finite volume method [11] and the above proposed approach. Due to the axial symmetry of the system under consideration the cylindrical coordinate system was used. It is assumed that the axis $O z$ coincides with the axis of the rod 1 and perpendicular to the ground surface 2 (see Fig. 3). It is also assumed that the calculation area is limited by a rectangle with sides $z=0, z=Z_{\max }, r=R_{\max }$, $r=0$ (see Fig. 3) and is divided into elementary rectangular cells.

In order to reduce the calculation region on its boundaries the so-called well-matched layers (PML) [6] (see 3 and 4 in Fig. 3) are introduced. These layers perform a supporting role and are need for guarantee the rapid and reflectionless decrease of the distortion of the EF caused by the presence of the considered objects when approaching the calculation region's boundaries.

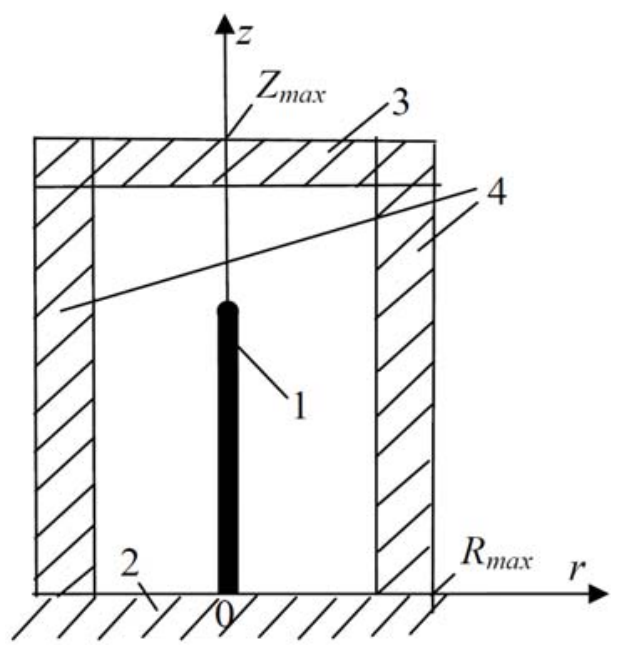

Fig. 3. Section $z=0$ of the considered calculation system, including conductive rod. 1 - rod; 2 - ground; 3, 4-PML

Distribution of the EF in the PML is not included in the results of the calculation. It is supposed that the electrical conductivity in this layer of thickness $d$ is a tensor, has different values in the directions of the coordinate axes $\mathrm{Or}, \mathrm{Oz}$ and varies in depth of the layer according to the polynomial law. Thus, for PML, which is perpendicular to the axis Or (see 4 in Fig. 3), changing 
the $r$-th $-\gamma_{r}^{P M L}(r)$ and the $z$-th $-\gamma_{z}^{P M L}(r)$ components of the conductivity tensor in the direction of the axis $\mathrm{Or}$ is written as [6]:

$$
\gamma_{r}^{P M L}(r)=\gamma_{0} \cdot k_{r}(r), \gamma_{z}^{P M L}(r)=\gamma_{0} / k_{r}(r),
$$

where $k_{r}(r)=1+\left(k_{\max }-1\right) \cdot(r / d)^{m} ; k_{\max }$ is the maximal value of $k_{r}$ on the external boundary of the PML-layer; $m$ is the power; $\gamma_{0}$ is the value of the conductivity of the medium adjacent with internal boundary of the PML.

Values of the conductivity tensor components in PML which is perpendicular to the axis $O r$ (see 4 in Fig. 3) are the following:

$$
\gamma_{z}^{P M L}(z)=\gamma_{0} \cdot k_{z}(z), \quad \gamma_{r}^{P M L}(z)=\gamma_{0} / k_{z}(z),
$$

where $k_{z}(z)=1+\left(k_{\max }-1\right) \cdot(z / d)^{m}$.

The values of electric conductivity in the areas of PML-layers crossing are found by multiplication of the respective components in each of the layers.

The conditions at the boundaries of the calculation area (see Fig. 3) are the following: $\varphi=0$ on the ground surface $(z=0) ; \partial \varphi / \partial n=0$ : at $R=0$ and at $r=R_{\max }$ (on the external boundary of the calculation area). In order to take into account that on the upper boundary of the calculation after the PML-layer the intensity of the applied EF equals to $E_{0}$, at $z=z_{\max }$ the condition $\partial \varphi / \partial n=k_{\max } \cdot E_{0}$ is used (for details, see [8]).

Comparison with analytical solution for a sphere in the homogeneous EF is showed that using ten layers of PML with parameters $m=5, k_{\max }=500 \cdot \gamma_{0}$ the relative error of calculation of intensity and potential of the EF no more than $3 \%$ is provided.

The influence of the geometry of rods with rounded tops on the maximum intensity of the EF. In order to assess the effect of height of rods on the possibility of corona discharge development from them distributions of the EF at different heights of rods were calculated.

Preliminary calculations in sequential increasing size of the limited by PML calculation area in the direction of the coordinate axes, as well as reducing the computational mesh's step have been carried out. It was believed that the solution adequately describes the distribution of the EF, when the values of intensities and potentials stop to change at sequential twice reduction of the step of the calculation grid and increasing its size. An analysis of the data made the following conclusions: so that the relative error does not exceed $3 \%$, size of the calculation area in the radial direction should be not less than the height of the $\operatorname{rod}(H)$ and in the vertically - in 1.2 times more than $H$; the step of the calculation grid mesh be no more than $R / 10$ (where $R$ is the radius of rod rounding).

The case is considered when it can be supposed that the uniform EF is applied to the rod. According to the literature (see, for example, [12, p. 188 and further]) at the discharges in systems «rod - plane» a so-called critical radius of the rod is. It is determined by the condition of the identity of the character of rupture in systems with rods, radius of which is less than the critical radius: $R \leq R_{c r}$, because at this condition the breakdown voltage does not depend on the value of $R$ because of the corona. The case where the value of the radius of the rod equals to the critical radius is considered $-R=R_{c r}=0.1 \mathrm{~m}$ [12].

Fig. 4 shows the results of numerical calculation of the dependence of $E_{\max }$ - maximal intensity levels of the $\mathrm{EF}$ in the vicinity of the conductive rods tops located in the external uniform field of strength $E_{0}$ (see curve 1) on their height $H$. The calculation was performed at the following of the calculation mesh: $\Delta_{r}=\Delta_{z}=R / 10$, $Z_{\max }=1.2 \cdot H, R_{\max }=H, H=v a r$.

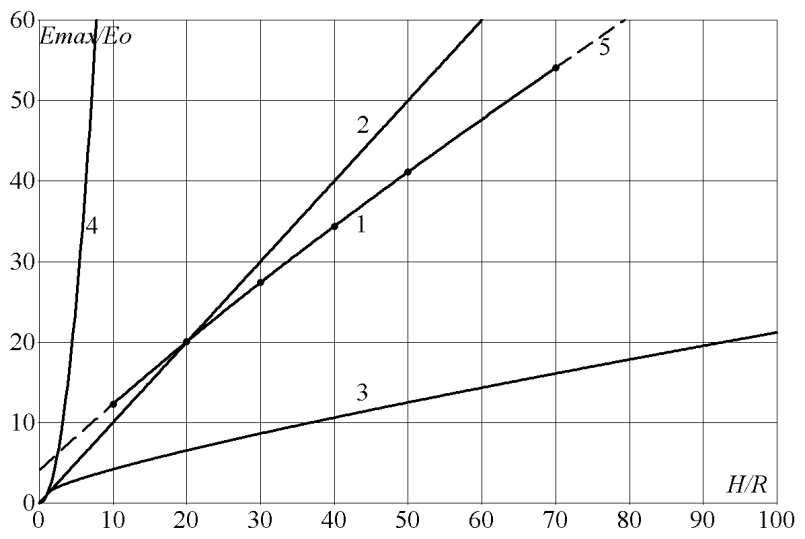

Fig. 4. Dependence of the maximal intensity of the EF in the vicinity of the conductive rod on its height $H: 1$ - numerical calculation by using the described approach; 2 - dependence $E_{\max }=E_{0} H / R ; 3,4$ - analytical solutions for conductive prolate ellipsoid at a distance from the top $l=0$ (3) and $l=R=0.1 \mathrm{~m}$ (4);

5 - polynomial approximation of the curve 1

The Figure also presents results of engineering estimates for $E_{\max }$ (curves $2-4$ ). Curve 2 corresponds to the assessment of $E_{\max }$ as ratio of the potential of the EF at the height of the top of the grounded rod to its radius $(R)$ :

$$
E_{\text {max }}=E_{0} \cdot H / R .
$$

Curves 3 and 4 are obtained as a result of the use of analytical expression for the intensity of the EF above the ellipsoid on its axis (see, for example, [13]) at a distance from its top $\Delta=R_{c r}$ (curve 3 ) and $\Delta=0$ (curve 4). As can be seen from a comparison of curves $1-4$, the values $E_{\max }^{*}=E_{\max } / E_{0}$ obtained with real shape of the rod (cylinder whose vertex is rounded) occupy an intermediate position relative to the smallest levels (curve 3 ) that correspond to the distance from the rod which is equal to critical radius $R_{c r}$ and maximal levels that match a point on the top of the equivalent ellipsoid (curve 4). In Fig. 4 curve 5 shown by a dotted line corresponds to a polynomial approximation of the curve 1 .

For the range of the change of the ratio of the height of core $(H)$ to its top rounding radius $(R)$ within $10<H / R<70$ the calculated curve of the dependence $E^{*}(H / R)$ can be approximated by a more simple function with which for object which is in the area of the electric 
field impact with intensity $E_{0}[\mathrm{~V} / \mathrm{m}]$ it is possible to find a boundary value for the ratio of the radius of rounding of its top to its height, which ensures guarantees suppression of the corona at this object:

\section{Conclusions.}

$$
R / H>0.7 /\left(3 \cdot 10^{6} / E_{0}-6.08\right) \text {. }
$$

1. The proposed method of taking into account the curved interface between media based on the finite volume method utilization permits to calculate the electric field distribution on the surface of the sphere and in its vicinity with relative error not exceeding $3 \%$, at the step of the calculation mesh not more than $0.1 \cdot R$ (where $R$ is the radius of the sphere).

2. Using the proposed method of taking into account the curved interface between media dependences of maximum electric field strength at the tops of the rods on the ratio between their diameter and height are calculated.

3. An expression to assess by which radius they should round off the sharp edges on objects of the height $H$, located in the area of the EF action of intensity $E_{0}$ so that they are not there corona discharge.

4. It is shown that the use of analytical solutions for conductive prolate ellipsoid leads to substantial error at placement of levels of maximum intensity near the tops of the rods.

\section{REFERENCES}

1. Toal B., McMillen M., Murphy A., Atkinson R., Pollard R. Tuneable magneto-optical metamaterials based on photonic resonances in nickel nanorod arrays. Materials Research Express, 2014, no.1, pp. 1-11. doi: 10.1088/20531591/1/1/015801.

2. Bazelian E.M., Razhanskii I.M. Iskrovoi razriad v vozdukhe [Spark discharge in air]. Novosibirsk, Nauka Publ., 1988. 165 p. (Rus).

3. Samarskii A.A. Teoriia raznostnykh skhem [Theory of difference schemes]. Moscow, Nauka Publ., 1989. 616 p. (Rus)

4. Popov E., Nevière M., Gralak B., Tayeb G. Staircase approximation validity for arbitrary-shaped gratings. Journal of the Optical Society of America A, 2002, vol.19, no.1, pp. 33-42. doi: 10.1364/josaa.19.000033.

5. Gjonaj E., Lau T., Schnepp S., Wolfheimer F., Weiland T. Accurate modeling of charged particle beams in linear accelerators. New Journal of Physics, 2006, no.8, pp. 1-21. doi: $\mathbf{1 0 . 1 0 8 8 / 1 3 6 7 - 2 6 3 0 / 8 / 1 1 / 2 8 5 . ~}$
6. Taflove A., Hagness S. Computational electromagnetics: the finite difference time domain method. Boston - London: Artech House, 2000. - 852 p.

7. Rezinkina M.M. The calculation of the penetration of a lowfrequency three-dimensional electric field into heterogeneous weakly conducting objects. Elektrichestvo - Electricity, 2003, no.8, pp. 50-55. (Rus).

8. Rezinkina M.M. Numerical calculation of the magnetic field and magnetic moment of ferromagnetic bodies with a complex spatial configuration. Technical Physics, 2009, vol.54, no.8, pp. 1092-1101. doi: 10.1134/S1063784209080027.

9. Tamm I.E. Osnovy teorii elektrichestva [Bases of the theory of electricity]. Moscow, Nauka Publ., 1989. 504 p. (Rus).

10. Stretton Dzh.A. Teoriia elektromagnetizma [Theory of electromagnetism]. M.-L.: OGIZ, Gostekhizdat Publ., 1948. 539 p. (Rus).

11. Patankar S. Chislennye metody resheniia zadach teploobmena $i$ dinamiki zhidkosti [Numerical methods of solution of problems of heat exchange and dynamics of liquid]. Moscow, Energoatomizdat Publ., 1984. 150 p. (Rus).

12. Cooray V. Lightning Protection. London: The Institution of Engineering and Technology, 2010. 1036p.

13. Kuchinskii G.S., Kizevetter V.E., Pintal' Iu.S. Izoliatsiia ustanovok vysokogo napriazheniia [Isolation of installations of high tension]. Moscow, Energoatomizdat Publ., 1987. 368 p. (Rus).

Received 29.10.2015

\section{E.I. Sokol ${ }^{1}$, Doctor of Technical Science, Professor,} Corresponding Member of the National Academy of Science of Ukraine,

M.M. Rezinkina ${ }^{2}$, Doctor of Technical Science,

E.V. Sosina ${ }^{1}$, Postgraduate Student,

O.G. Gryb ${ }^{1}$, Doctor of Technical Science, Professor,

${ }^{1}$ National Technical University «Kharkiv Polytechnic Institute», 21, Frunze Str., Kharkiv, 61002, Ukraine.

e-mail: elenasosina09@gmail.com

${ }^{2}$ State Institution «Institute of Technical Problems of Magnetism of the NAS of Ukraine», 19, Industrialna Str., Kharkiv, 61106, Ukraine. e-mail:marinar2@mail.ru

\section{How to cite this article:}

Sokol E.I., Rezinkina M.M., Sosina E.V., Gryb O.G. Numerical computation of electric fields in presence of curvilinear interface between conductive and non-conductive media. Electrical engineering \& electromechanics, 2016, no.1, pp. 42-47. doi: 10.20998/2074-272X.2016.1.08. 